\section{Cyclic stretch of the substratum using a shape-memory alloy induces directional migration in Dictyostelium cells}

\author{
Yoshiaki Iwadate ${ }^{1,2}$ and Shigehiko Yumura ${ }^{1}$ \\ ${ }^{1}$ Department of Functional Molecular Biology, Graduate School of \\ Medicine, Yamaguchi University, Yamaguchi, Japan and 2PRESTO, Japan \\ Science and Technology Agency, Saitama, Japan
}

BioTechniques 47:757-767 (September 2009) doi 10.2144/000113217

Keywords: cell polarity; cell system; mechanosensing; cell migration

Supplementary material for this article is available at www.BioTechniques.com/article/113217.

Living cells are constantly subjected to various mechanical stimulations. They must sense the mechanical aspects of their environment and respond appropriately for proper cell function. In general, cells adhere to substrata. Thus, the cells must receive and respond to mechanical stimuli mainly from the substrata. For example, migrating cells can create their own polarity and migrate in a certain direction even in the absence of any attractive substance. In order to generate such polarity, cells must sense mechanical stimuli from the substrata and transduce these stimuli into intracellular signals. To investigate the relationship between signals derived from mechanical stimuli and related cell functions, one of the most commonly used techniques is the application of mechanical stimuli via stretching of elastic substrata. Here, we developed a new stretching device using a shape-memory alloy (SMA). An SMA has three advantages as an actuator of stretching devices: $(i)$ the cost of the SMA required for the device is inexpensive, $\sim \$ 30$ USD, (ii) the size of an SMA is very small (0.62 $\mathrm{mm}$ in diameter and $22 \mathrm{~mm}$ in length), and (iii) an SMA does not generate any vibrating noise, which can negatively affect cells. In response to the cyclic stretching by the new stretching device, Dictyostelium discoideum cells migrated perpendicular to the stretching direction and the migrating speed increased significantly. To our knowledge, this is the first report indicating that migrating cells can create their own polarity by the mechanical stimuli from the substrata.

\section{Introduction}

Living cells are constantly subjected to a wide variety of mechanical stimulations such as shear flow and substratum strain. They must sense the mechanical aspects of their environment and respond appropriately for proper cell function. For example, blood shear flow activates various cell functions in vascular endothelial cells, such as gene expression, proliferation, and apotosis (1). In the auditory hair cells of vertebrates, stereovilli deflections open mechano-electrical transduction channels and cause changes in the membrane potential (2). To understand the mecha- nisms of the mechanosensing system in cells, artificial mechanical stimuli-such as sucking of a cell portion using a micro pipette (3-6), hydrodynamic shear-flow (7-9), and manipulation of microbeads attached to a cell with magnetic $(10,11)$ or optical tweezers $(12,13)$ - have been applied (14), leading to many findings, such as the existence of signaling pathways triggered by mechanical stimuli (15).

In general, cells adhere to the substratum via focal adhesion sites. Thus, it seems that the cells receive mechanical stimuli mainly from substrata in the physiological condition $(15,16)$. To mimic this situation, one of the most appropriate techniques for applying mechanical stimuli artificially is to stretch the elastic substratum to which cells adhere. In response to the cyclic stretching of the elastic substratum, intracellular stress fibers in endothelial cells are rearranged perpendicular to the stretching direction $(17-20)$

Fast-moving cells, such as Dictyostelium discoideum cells, can create their own polarity and migrate in a certain direction even in the absence of any chemoattractant. In order to generate polarity without any chemoattractant, it is presumed that cells sense mechanical inputs from the substratum. To investigate the relationship between substratum mechanical inputs and migration direction, a device for cyclically stretching the substratum could be a powerful tool. However, migration of fastmoving cells has not, to our knowledge, been observed under cyclic stretching.

A shape-memory alloy (SMA) is a material that remembers its own geometry. After an SMA has been deformed from its original crystallographic configuration, it regains its original geometry by itself during heating. SMA is an appropriate material for the actuator of the stretching device because of the following three reasons: (i) the cost of the SMA required for the device is low, $\sim$ \$30 USD; (ii) the size required for an SMA for stretching the elastic substratum is very small $(0.62 \mathrm{~mm}$ in diameter and 22 $\mathrm{mm}$ in length), enabling a compact setup (including electrical circuit) which is also advantageous for use with a microscope stage; and (iii) an SMA does not generate any vibrating noise when it moves.

Here, we developed a new stretching device using an SMA as an actuator. Using this new device, we applied cyclic stretching stimuli to migrating Dictyostelium cells. Interestingly, in response to the cyclic stretching, the cells migrated perpendicular to the stretching direction and the migrating speed of the cells increased significantly. To our knowledge, this is the first report indicating that the migrating cells can create their own polarity by the mechanical stimuli from the substrata.

\section{Materials and methods}

The overall setup for the new cell stretching device is shown in Figure 1A. The device is composed of an elastic sheet, coiled SMAs, a cooling fan, and an electrical circuit to produce voltage pulses for cyclic contraction of the SMAs.

Preparation of elastic sheets

To apply stretching stimuli to cells via the attached substratum and observe their 
A

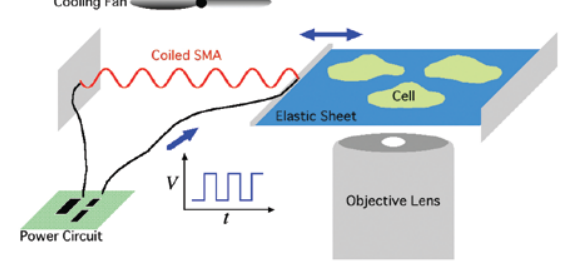

B

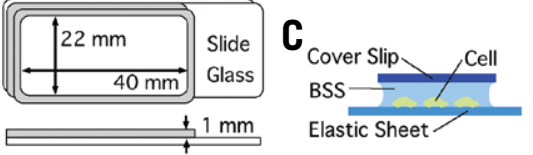

D

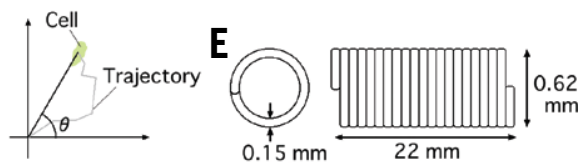

F

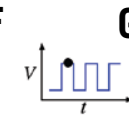

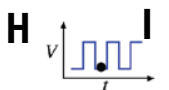

J

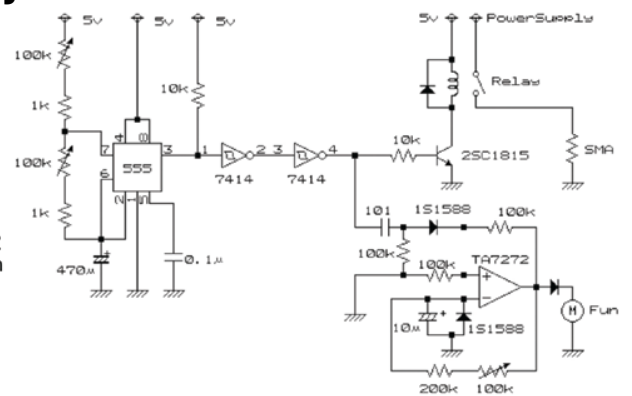

K

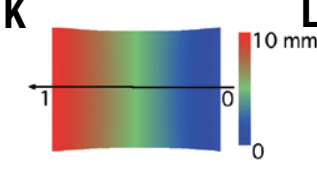

M
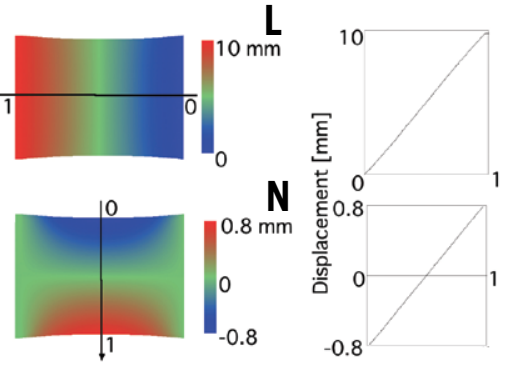

0

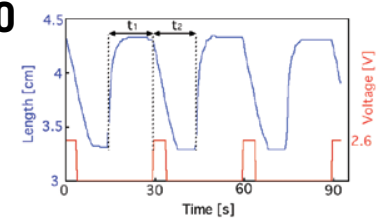

Figure 1. Setup of the cell stretching device using coiled SMA. (A) The cell stretching device was composed of an elastic sheet, 4 pieces of coiled SMA, a fan for cooling the SMA, and an electric power circuit to produce sequential voltage pulses (graph, blue single-headed arrow) for cyclic shrinkage of the SMAs (blue double-headed arrow). Four pieces of coiled SMA were connected in parallel, although only one coiled SMA is drawn in the figure. The right side of the sheet and the left side of the SMA were fixed. The device was placed on the stage of an inverted microscope. Dictyostelium cells dispersed on the elastic sheet were observed through a $20 \times$ objective lens. (B) The mold to solidify elastic sheets, made from a slide glass with an acrylic frame (gray). Top: top view. Bottom: side view. Elastic sheets were made from a kit of platinum-catalyzed liquid silicone composed of a silicon rubber base and a curing agent. (C) A droplet containing Dictyostelium cells on the elastic sheet was covered with a $7 \mathrm{~mm} \times 7 \mathrm{~mm}$ coverslip to prevent light scattering at the rounded surface of the droplet. BSS, Bonner's standard saline; see "Cell culture" section of "Materials and methods" for details. (D) Definition of the migration direction for statistical analysis of the relationship between the cyclic stretching stimuli and cell migration direction. The migrating angle $(\theta)$ of a cell was defined as that between a horizontal line and a straight line connecting the starting and ending points of a migrating cell during a single experiment. (E) Schematic of a coiled SMA used as an actuator in the new stretching device. Left: front view. Right: lateral view. (F-I) Drive for the cyclic stretching of the elastic sheet. Panels F and G: when the voltage between the two ends of the coiled SMA was high (black dot in panel F), the SMA shrank and the sheet stretched (panel G). Panels $\mathrm{H}$ and I: when the voltage was low (black dot in panel $\mathrm{H}$ ), the sheet shrank due to its own elasticity and the SMA stretched (panel I). The duty ratio of shrinkage and stretch was adjusted to 1:1 by modulating the duty ratio of the square voltage pulses. When the time cycle is shorter than $20 \mathrm{~s}$, the fan in panel A was used for cooling the SMA to release the contractile force quickly. Lengths of the sheet on the central perpendicular lines (I and $\left.I^{\prime}\right)$ in the sheets in panels $\mathrm{G}$ and I were measured for the estimation of Poisson's effect in Table 1. $(\mathrm{J})$ Electric power circuit to generate sequential square voltage pulses and control the cooling fan which consist essentially of an analog timer IC, NE555, and a power operational amplifier IC, TA7272. (K-N) Homogeneity of the deformation of the elastic sheet clamped in the new device. Displacements of the sheet in the stretching direction (panel $\mathrm{K}$ ) and in the perpendicular direction (panel M) are calculated by FEA of the plane stress state and visualized as pseudo-color images. The original lengths of horizontal and perpendicular directions were $33 \mathrm{~mm}$ and $20 \mathrm{~mm}$, respectively. The displacements of the sheet along the arrows in panels $\mathrm{K}$ and $\mathrm{M}$ were plotted in panels $\mathrm{L}$ and $\mathrm{N}$, respectively. The numbers 0 and 1 in panels $K$ and $M$ indicate the positions 0 and 1 in panels $L$ and $N$, respectively. (O) Kinetic property of the substratum. Red line: sequential square voltage pulses with a peak value of $2.6 \mathrm{~V}$ were applied to the SMA. Blue line: cyclic stretching of the substratum caused by the electric application. The duty ratio $\left(t_{1}: t_{2}\right)$, was fixed at $1: 1$ by adjusting the durations of the $2.6-\mathrm{V}$ and $0-\mathrm{V}$ applications to $4 \mathrm{~s}$ and $26 \mathrm{~s}$, respectively.

response, an elastic sheet with optimal elasticity and clearness was prepared as a substratum. We selected an elastomeric polydimethylsiloxane (PDMS) kit (Sylgard 184, Dow Corning Toray, Tokyo, Japan) of Young's modulus $2.2 \pm 0.1 \mathrm{MPa}(21)$ as the material for the elastic sheet. The PDMS kit contains two parts, a liquid silicon rubber base and a curing agent, and uses a platinum complex to participate in hydrosilylation of a vinyl functional siloxane polymer by a hydride functional siloxane polymer. The silicone elastomer made from the kit is clear and colorless. A silicon rubber base and a curing agent were mixed at a 10:1 ratio by weight. A 300-mg aliquot of the mixture was poured into a $22 \mathrm{~mm} \times 40 \mathrm{~mm} \times 1 \mathrm{~mm}$ mold made from a slide glass (Cat. no. S-2441, Matsunami, Osaka, Japan) with an acrylic frame (Figure 1B). The mixture in the mold form was allowed to solidify at room temperature $\left(23^{\circ} \mathrm{C}\right)$ for 2 days. The solidified sheet was peeled off carefully (to facilitate this, the mold was previously prepared with a thin layer of only the silicon rubber base, before the mixture was added). The thickness of the sheet, except for the periphery, was $\sim 200 \mu \mathrm{m}$. The periphery was slightly thicker because of the surface tension of the liquid silicone.

\section{Cell culture}

Cells of the Dictyostelium discoideum cAR1/cAR3 [receptors of chemoattractant (cAMP)] double-mutant cell line RI9 (22) were cultured in HL5 medium $[1.3 \%(\mathrm{w} / \mathrm{v})$ bacteriological peptone, $0.75 \%$ (w/v) yeast extract, $85.5 \mathrm{mM} \mathrm{D}$-glucose, 3.5 $\mathrm{mM} \mathrm{Na}_{2} \mathrm{HPO}_{4} 12 \mathrm{H}_{2} \mathrm{O}, 3.5 \mathrm{mM} \mathrm{KH}_{2} \mathrm{PO}_{4}$, $\mathrm{pH}$ 6.4] and developed in Bonner's standard saline (BSS, $10 \mathrm{mM} \mathrm{NaCl}, 10 \mathrm{mM} \mathrm{KCl}$, $3 \mathrm{mM} \mathrm{CaCl}_{2}$ ) for $>12 \mathrm{~h}$ at $4^{\circ} \mathrm{C}$.

\section{Microscopy}

One hundred microliters of cell suspension was settled on the elastic sheet. A $7 \mathrm{~mm} \times 7 \mathrm{~mm}$ coverslip (Cat. no. 1;
Matsunami, Osaka, Japan) was floated on the droplet to prevent light scattering at the rounded surface of the droplet and improve the observed images (Figure 1C).

Migrating cells plated on the elastic sheet were observed under phase-contrast microscopy using an inverted microscope (TMS, Nikon, Tokyo, Japan) with a $20 \times$ objective lens (20/0.4, Nikon, Tokyo, Japan). Images taken by a CCD camera (model no. XC-EI50, Sony, Tokyo, Japan) were transferred to a computer (Macintosh G4, Apple Japan, Tokyo, Japan) at 30-s intervals through a frame grabber board (LG3, Scion, Frederick, MD, USA) and analyzed using ImageJ Ver 1.40 (http:// rsbweb.nih.gov/ij) with two plug-ins: Manual Tracking, programmed by Fabrice Cordelieres (Institut Curie, Orsay, France), and a Chemotaxis and Migration Tool, programmed by Gerhard Trapp and Elias Horn (Ibidi, Martinsried, Germany). In this analysis, the angle ( $\theta$ in Figure 1D) between the horizontal line and the 
A
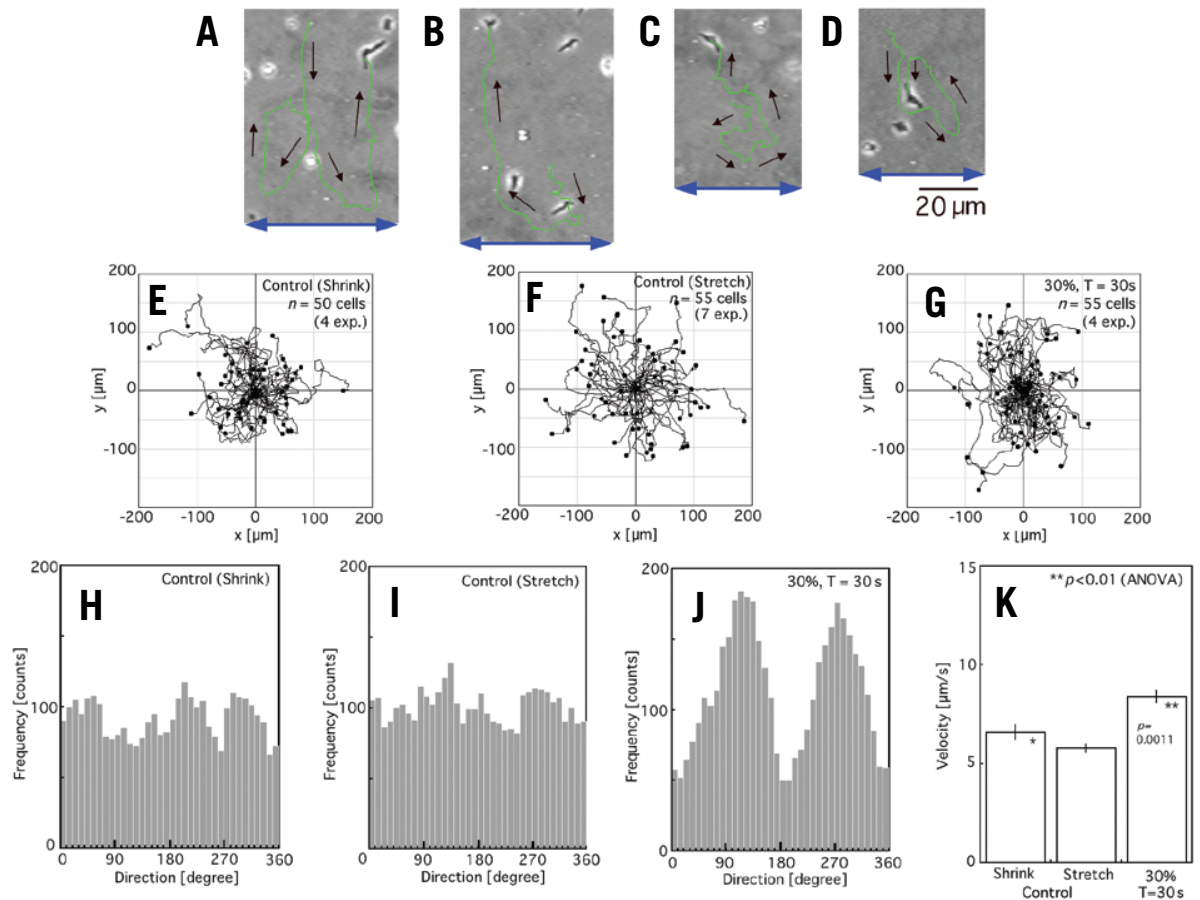

straight line which connected the starting and ending points of a migrating cell was measured. As an index of the directional migration, $|\sin \theta|$ was calculated. When a cell migrates parallel to the stretching direction, the value should be 0 . On the other hand, when a cell migrates perpendicularly, the value should be 1 . Frequencies of $\theta$ are shown as histograms in Figures 2 and 3 . The migrating speed was calculated by dividing the total length of the trajectory by total time. One-way analysis of variance (ANOVA) and multiple comparisons of means (Ryan's method) were applied to examine the differences among the data.

Cyclic stretch of the silicone sheet

To stretch the elastic sheet, we used a coiled SMA (BioMetal Helix150, Toki Corp., Tokyo, Japan) as an actuator. The diameters of the fiber and the coil are 0.15 and $0.62 \mathrm{~mm}$, respectively (Figure 1E). The SMA has two interesting characteristics: $(i)$ after expansion by mechanical force, it contracts and recovers its original shape with the application of heat, and (ii) it demonstrates electric resistance. Thus, the SMA can produce heat by itself according to Joule's law: $P=V^{2} / R$, where $P$ is the power (watts), $V$ is the potential difference (volt) between the two ends of a piece of SMA, and $R$ is the resistance (ohms) of the SMA. Thus, in response to the application of voltage pulses to the expanded SMA, it contracts due to the self-generated heat. A coiled SMA can be expanded to $>2 \times$ its original length.

A piece of coiled SMA was connected to one side of the elastic sheet via an acrylic part to prevent heat transfer from the SMA to the sheet and the other side of the sheet was fixed (Figure 1, A, F-I). Sequential voltage square pulses were applied to the coiled SMA (blue single-headed arrow, Figure 1A), which induced cyclic stretching and shrinkage of the elastic sheet (blue doubleheaded arrow, Figure 1A). When the voltage between the two ends of the coiled SMA was high (black dot, Figure 1F), the SMA shrank and the sheet stretched (Figure 1G). On the other hand, when the voltage was low (black dot, Figure 1H), the sheet shrank due to its own elastic force (Figure 1I).

Voltage pulses were applied using a simple self-made electric power circuit (Figure 1J). An astable multi-vibrator circuit with NE555 (Texas Instruments, Dallas, TX, USA) was used as a source of voltage pulses. When the time cycle was shorter than $20 \mathrm{~s}$, a fan (Figure 1, A and J) was used for cooling the SMA to release the contractile force quickly. The time of the cooling was optimized by a one-shot multi-vibrator circuit with a power operational amplifier, TA7272 (Toshiba, Tokyo, Japan).

The silicone sheet and SMA were settled in an acrylic frame. The frame was made from an acrylic board using a NC milling machine (COBRA 2520; Original Mind, Nagano, Japan). The costs of the electric circuit and the acrylic frame were $<\$ 50$ USD and $<\$ 20$ USD, respectively. It took less than 1 day to make the electric circuit and the acrylic frame.

Finite element analysis

We estimated the Poisson's effect and the homogeneity of the deformation of the
Figure 2. Directional migration of Dictyostelium cells in response to the cyclic stretching. (A-D) Phase-contrast images of typical migrating cells with representative tracking (green lines) of cells under cyclic stretching stimuli. The stretching directions of the substrata and the migrating directions of the cells are indicated by blue double-headed arrows and black single-headed arrows, respectively. Time cycle and stretching ratio were $30 \mathrm{~s}$ and $30 \%$, respectively. (E-G) Superimposed trajectories of migrating cells on the shrunken sheet without cyclic stretching (panel $E ; n=50$ from four experiments), on the 30\% stretched sheet without cyclic stretching (panel F; $n=55$ from seven experiments), and under cyclic stretching (panel G; $n=55$ from four experiments). ( $\mathrm{H}-\mathrm{J}$ ) Frequencies of the cell migrating direction $(\theta)$ calculated from panels $E-G$, respectively. (K) Averaged speeds of cell migrations. Left: on the shrunken sheet without cyclic stretching. Center; on the $30 \%$ stretched sheet without cyclic stretching. Right: under cyclic stretching. Bars, SEM. The speed of cell migration under cyclic stretching $\left({ }^{* *}\right)$ was significantly higher than that on the shrunken sheet without cyclic stretching $(*, P<0.01$, ANOVA).

elastic sheet clamped in the stretching device by finite element analysis (FEA) of the plane stress state. FEA was performed by using VisualFEA software (Intuition Software, Chonbuk, South Korea).

\section{Results and discussion}

In elastic sheets, shortening of the sheet perpendicular to the axis of stretch (Poisson's effect) must take place. First, characteristics of the elastic sheet clamped in the new device were determined (Table 1). The change of the length of the sheet indicated as the central perpendicular lines $\left(l\right.$ and $l^{\prime}$ in Figure 1, G and I) by stretching to the horizontal axis was measured. In the range of $5-30 \%$ stretching, the ratio of perpendicular shrinkage was $1 / 4$ of the stretching length. This value agrees well with the theoretical value calculated by FEA of the plane stress state, in which Young's modulus and Poisson's ratio were 2.2 MPa and 0.3, respectively. Thus, we assumed that the sheet clamped in the new device behaved as an elastic sheet in which the Poisson's ratio was 0.3 . Then, the deformation of the sheet was simulated by FEA (Figure 1, K-N). Figure $1 \mathrm{~K}$ and $1 \mathrm{M}$ show the pseudo-color images of the displacements of each point of the sheet in the stretching direction (Figure $1 \mathrm{~K}$ ) and in the perpendicular direction (Figure $1 \mathrm{M})$, respectively. In this calculation, $30 \%$ stretching was applied. The displacements of the sheet along the arrows in Figure $1 \mathrm{~K}$ and $1 \mathrm{M}$ were plotted in Figure $1 \mathrm{~L}$ and $1 \mathrm{~N}$, respectively. The numbers 0 and 1 in Figure $1 \mathrm{~L}$ and $1 \mathrm{~N}$ indicate the positions 0 and 1 in Figure $1 \mathrm{~K}$ and $1 \mathrm{M}$, 
Table 1. Characteristics of the elastic sheets clamped in the new stretching device

\begin{tabular}{|c|c|c|c|c|}
\hline \multicolumn{2}{|c|}{ Parallel to stretching direction } & \multicolumn{3}{|c|}{ Perpendicular to stretching direction } \\
\hline Length [mm] & Stretch ratio [\%] & Lengtha $[\mathrm{mm}]$ & Shrinkage Ratio [\%] & Theoretical length ${ }^{\mathrm{b}}[\mathrm{mm}]$ \\
\hline 33 & 0 & 20 & 0 & - \\
\hline 36 & 10 & 19.5 & 2.5 & 19.8 \\
\hline 40 & 20 & 19 & 5 & 19 \\
\hline 43 & 30 & 18.5 & 7.5 & 18.3 \\
\hline
\end{tabular}

aLengths of the central perpendicular lines ( $\mathrm{I}$ and $\left.\mathrm{I}^{\prime}\right)$ of the sheets in Figure $1(\mathrm{~F}$ and $\mathrm{H})$ were measured. bTheoretical length was calculated by FEA of the plane stress state, in which Young's modulus and Poisson's ratio were $2.2 \mathrm{MPa}$ and 0.3 , respectively.

respectively. As shown in Figure 1L and $1 \mathrm{~N}$, the displacements increase linearly from position 0 to 1 , suggesting that the mechanical stimulation from the sheet to the cell is uniform throughout the sheet.

To generate an enough force to stretch the elastic sheet, 4 pieces of coiled SMA, $2.2 \mathrm{~cm}$ in length, were connected in parallel to the edge of the sheet. The total resistance of the 4 pieces of SMA was $2.6 \mathrm{ohms}$. Sequential square voltage pulses with a peak value of $2.6 \mathrm{~V}$ were applied to the SMA. This electric application caused cyclic stretching of the elastic sheet. The duty ratio and the time cycle of the cyclic stretching were easily controlled by changing the durations of the 2.6 and $0 \mathrm{~V}$ applications. The duty ratio was fixed at 1:1 throughout the experiments. The minimum time cycle of the new stretching device was $<5 \mathrm{~s}$. This is comparable in speed to commercially available stretching devices. Typical motion of the device is exhibited in Supplementary Movie S1, in which the stretching ratio and the time cycle are 30\% and $30 \mathrm{~s}$, respectively. The kinetic property of the substratum is shown in Figure 10. The duty ratio $\left(t_{1}: t_{2}\right.$ in Figure 1O) was fixed at $1: 1$. Under this condition, no medium flow was confirmed by the observation of sunken small particles detached from the substratum (data not shown), which indicated that the influence of shear stress to the cells would be negligible.

The costs of the SMA required for making the new stretching device was $\sim$ \$30 USD. Thus, the total cost of the new device including the electric circuit and the acrylic frame was $<\$ 100$ USD. This cost is much lower than the price of commercially available products (e.g., FX-4000, Flexcell, NC, USA, and STB-150, Strex, Osaka, Japan; >\$10,000). Moreover, the new device has two other advantages: its small size and lack of vibrating noise. In preliminary experiments, we applied the cyclic stretching to the Dictyostelium cells using a motor-driven device (STB-150, Strex, Osaka, Japan). They often detached from the substratum by vibration from the motor because of their weak adhesiveness (data not shown).

Using the new stretching device, we examined whether Dictyostelium cells could sense mechanical stimulations applied through the substratum and whether this influenced migration direction. The cAR1/cAR3 double-mutant cell line was used in this experiment to prevent spontaneous chemotactic response. Dictyostelium cells, developed in BSS for $>12 \mathrm{~h}$ at $4^{\circ} \mathrm{C}$, were dispersed on the elastic sheet and their migrations were recorded during the application of cyclic stretching stimuli (30\% stretching ratio and $30 \mathrm{~s}$ time cycle; Supplementary Movie S2). As shown in the movie, the cells can be observed clearly

\section{New Microcalorimetry Products FROM}

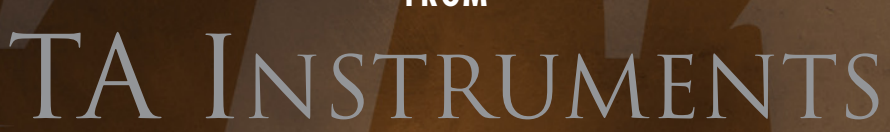

...The World Leader in Thermal Analysis, Rheology, and Microcalorimetry

NANO DSC

-High Sensitivity Scanning Calorimeter -Protein Denaturation, Stability

NANO ITC

-High Sensitivity Titration Calorimeter

-Protein Binding, Interactions, Kinetics

\section{TAM III}

-High Sensitivity Isothermal Calorimeter

-Stability, Compatibility, Morphology

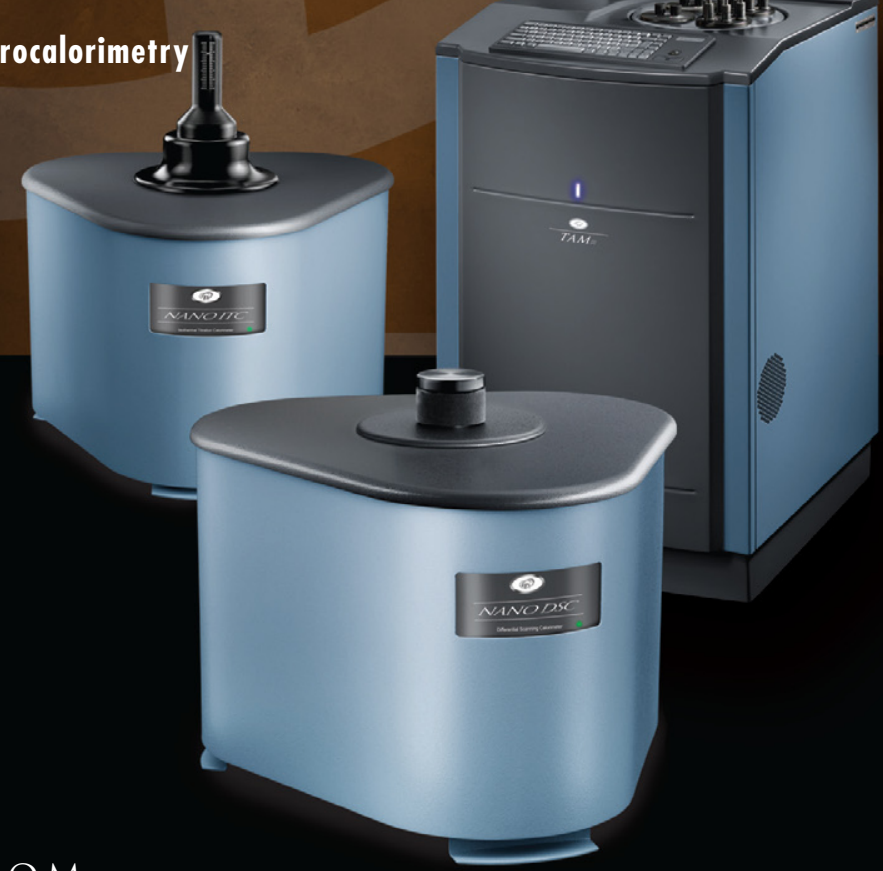


A
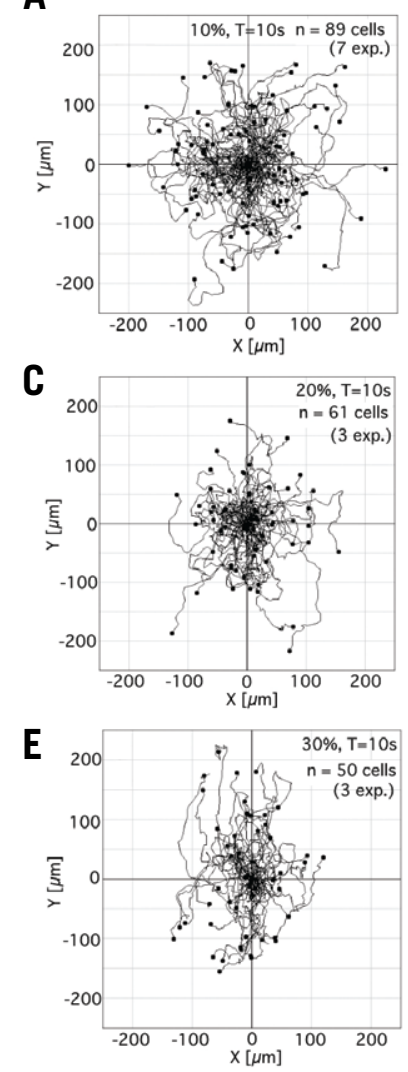

B
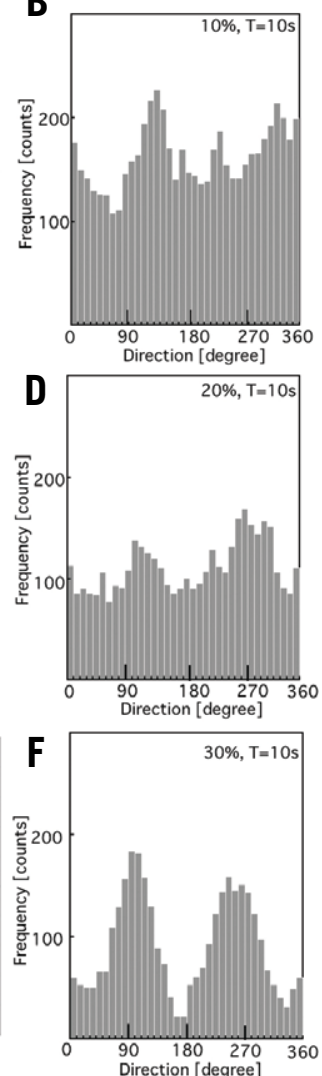

G

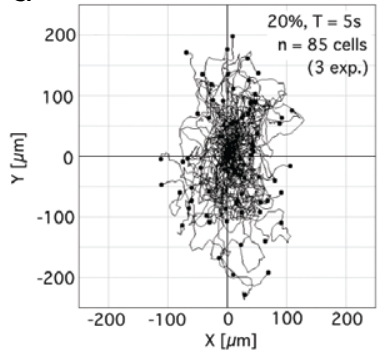

H

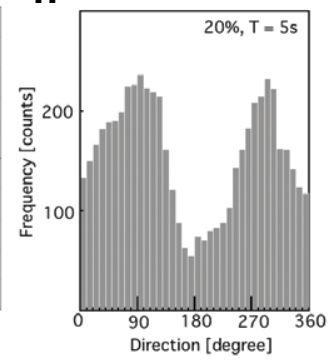

I
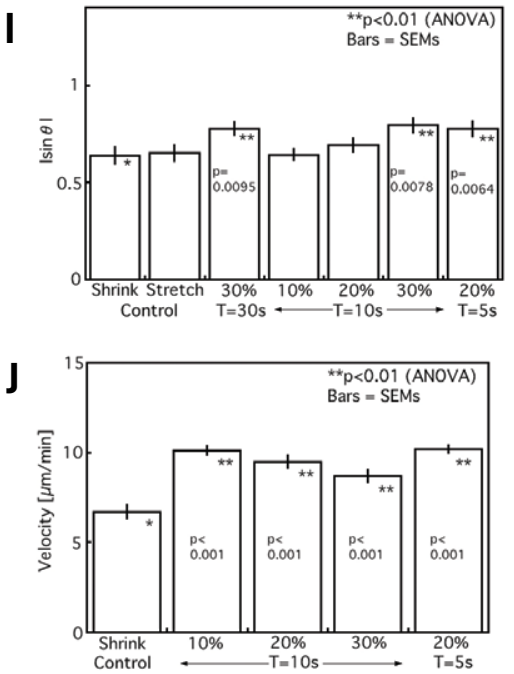

Figure 3. Directional migration of Dictyostelium cells in response to the cyclic stretching with different stretching ratios and time cycles. (A, $C$, E, and G) Superimposed trajectories of migrating cells under cyclic stretching. Stretching ratios and time cycles are indicated in each figure. (B, D, F, and $H$ ) Frequency of the cell migrating direction $(\theta)$ calculated from panels $A, C, E$, and $G$, respectively. (I) Averaged I $\sin \theta \mid$. When a cell migrates parallel to the stretching direction, the value should be 0 . On the other hand, when a cell migrates perpendicularly, the value should be 1 . The I $\sin \theta \mid$ values under several cyclic stretching stimuli with high stretching ratio and/or short time interval $\left(^{* *}\right)$ were significantly higher than that on the shrunken sheet without cyclic stretching $\left({ }^{*}, P<0.01\right.$, ANOVA). Bars, SEM. (J) Averaged speeds of cell migrations. The speeds of cell migrations under all cyclic stretching stimuli $\left.{ }^{* *}\right)$ were significantly higher than that on the shrunken sheet without cyclic stretching $\left({ }^{*}, P<0.01\right.$, ANOVA). Bars, SEM.

at the moment when the sheet shrinks. Thus, only the images from when the sheet shrank were extracted and accumulated sequentially (Supplementary Movie S3). From these sequential images, we analyzed the trajectory and velocity of the cells using plug-ins for ImageJ software (Figure 2). In Figure 2, cyclic stretching was applied horizontally $\left(0-180^{\circ}\right)$. Figure $2, \mathrm{~A}-\mathrm{D}$, shows typical trajectories of migrating cells (green lines) under the application of cyclic stretching. Stretching directions are indicated as blue double-headed arrows at the bottom of each figure. The cells tended to migrate perpendicular to the stretching direction $(n=50$, from four experiments). The cells migrated upward and downward with the same probability. Figure 2, G and J, shows the trajectories of migrating cells and the frequencies of cell migrating directions $(\theta)$, respectively. The migrations of the cells on the fixed sheets under the shrunken state (Figure 2, E and $\mathrm{H}$ ) and the stretched state (Figure 2, F and I) were observed as controls. As an index of perpendicular migration to the stretching direction, average $|\sin \theta|$ values in each condition were calculated ( 3 columns from the left in Figure 3I). The average migrating speeds of cells under the three conditions are summarized in Figure $2 \mathrm{~K}$.

From the $30 \%$ stretching, stiffening in the direction of stretching and softening in the perpendicular direction should take place due to the Poisson's effect. However, between the shrunken and $30 \%$ stretched sheets without cyclic stretching, there was no significant difference in $|\sin \theta|$ values $[0.64 \pm 0.042$, $\operatorname{SEM}(n=50)$ and $0.65 \pm 0.041(n=55)]$ or migrating speed $[6.7 \pm 0.038(n=50)$ and $5.8 \pm 0.20(n=55) \mu \mathrm{m} / \mathrm{min}$, Figures $2 \mathrm{~K}$ and $3 \mathrm{I}, P>0.01$, ANOVA]. These results indicate that the heterogeneous rigidity of the sheet did not induce directional cell migration. Thus, in the following experiments, we regarded the cell migration on the fixed shrunken sheet without cyclic stretching as a control where cells migrated randomly. When the significant difference of $|\sin \theta|$ (calculated by one-way ANOVA and Ryan's method) between a condition and the control was $<0.01$, we regarded the migration as directional.

When the cyclic stretching stimuli of $30 \%$ stretching ratio and $30 \mathrm{~s}$ time cycle were applied (Figure 2, G and J), $|\sin \theta|[0.78 \pm 0.031(n=55)]$ was significantly higher than that of the control $[0.64 \pm 0.042(n=50)$, Figure 3I, $P<0.01]$. The average migrating speed of cells with cyclic stretching $[8.2 \pm 0.31 \mu \mathrm{m} / \mathrm{min}$ $(n=55)]$ was significantly higher than that of control $[6.7 \pm 0.38 \mu \mathrm{m} / \mathrm{min}(n=50)$, Figure $2 \mathrm{~K}, P<0.01]$. These results clearly indicate that the Dictyostelium cells determine their migrating direction and increase their speed in response to the mechanical stimuli from the substratum.

Next, we examined the relationship between the stretching ratio and the degree of the directionality of the migration. Cell migrations under the cyclic stretching stimuli of 10-s time cycle and different stretching ratios $(10 \%, 20 \%$, and $30 \%)$ 
were analyzed (Figure 3, A-F, I). Figure 3, A, C, and E, shows the trajectories of migrating cells under each stretching ratio, respectively. Figure 3, B, D, and F, shows the frequencies of cell migrating direction. The $|\sin \theta|$ values in each condition are shown in Figure 3I. Under 10\% cyclic stretching stimuli, the cells migrated randomly (Figure 3, A and B). There was no significant difference in $|\sin \theta|$ between the $10 \%$ cyclic stretching stimuli $[0.64 \pm$ $0.029(n=89)]$ and the control condition $[0.64 \pm 0.042(n=50)]$. When $20 \%$ cyclic stretching was applied, two small peaks are shown at $90^{\circ}$ and $270^{\circ}$ in Figure 3D, although there is no significant difference in $|\sin \theta|$ between the $20 \%$ cyclic stretching stimuli $[0.69 \pm 0.036(n=61)]$ and the control $[0.64 \pm 0.042(n=50)$, Figure 3I]. Under the $30 \%$ cyclic stretching stimuli, the shape of the superimposed trajectories was vertically long (Figure 3E). Two clear peaks are shown at $90^{\circ}$ and $270^{\circ}$ in Figures $3 \mathrm{~F}$. The $|\sin \theta|$ under the $30 \%$ cyclic stretching stimuli $[0.80 \pm 0.037(n=50)]$ was significantly larger than that of the control $[0.64 \pm 0.042(n=50)$, Figure 3I, $P<0.01]$.

To examine the effect of time cycle, cell migrations under cyclic stretching stimuli of $20 \%$ stretching ratio and 5-s time cycle (Figure 3, G and $\mathrm{H}$ ) was compared with that of $20 \%$ stretching ratio and 10 -s time cycle (Figure 3, C and D). The shape of the superimposed trajectories was vertically long (Figure $3 \mathrm{G}$ ). The peaks at $90^{\circ}$ and $270^{\circ}$ in Figures $3 \mathrm{H}$ is clearly higher than that in Figure 3D. The value of $|\sin \theta|$ under cyclic stretching stimuli of $20 \%$ stretching ratio and 5 -s time cycle $[0.77 \pm 0.030(n=85)]$ was significantly larger than that of the control $[0.64 \pm 0.042(n=50)$, Figure 3I, $P<0.01]$.

On the other hand, the speeds of cell migrations under cyclic stretching stimuli of $10 \%, 20 \%$, and $30 \%$ stretching ratios and a 10 -s time cycles, and $20 \%$ stretching ratio and 5 -s time cycles $[10.1 \pm 0.25(n=89)$, $9.5 \pm 0.35(n=61), 8.7 \pm 0.34(n=50)$, $10.2 \pm 0.12(n=85) \mu \mathrm{m} / \mathrm{min},{ }^{* *}$ in Figure 1J] were significantly higher than that of the control $[6.7 \pm 0.38 \mu \mathrm{m} / \mathrm{min}(n=50)$, * in Figure 1J, $P<0.01]$.

Cells can perform persistent migration even in the absence of an external chemoattractant (23). In order to generate a polarity and migrate in a certain direction without any chemoattractant, cells presumably sense mechanical stimuli from the substratum. For example, fibroblasts sense rigidity of the substratum and move toward rigid areas (the so-called durotaxis) (24-26). To ascertain whether the migrating direction of cells is regulated by mechanical inputs from the substratum, detailed measurements of migrating cell trajectories during the application of continuous mechanical stimuli from the substratum are required [as is the case with chemotaxis (27) and galvanotaxis $(28,29)]$. In this study, the trajectory analysis of migrating cells under continuous mechanical stimuli from the substratum was performed with our new stretching device using SMA. This new device allowed us to demonstrate that mechanical stimuli influence the migration direction of the cells.

Using elastic sheets, it is difficult to exclude the Poisson's effect completely. In the case of our new device, when the sheet was stretched, $1 / 4$ of perpendicular shrinkage took place, simultaneously. Cells showed random migration under the cyclic stretching stimuli of $10 \%$ stretching ratio and 10-s time cycle (Figure 3, A, B, and I). This indicates that cyclic stretching of $<10 \%$ does not affect directionality of cell migration. In all experiments, the ratios of perpendicular shrinkage by Poisson's effect were $<7.5 \%$ (Table 1 ). Thus, the perpendicular shrinkage in all experiments should not affect the directionality of cell migration. On the other hand, the speed of cell migration increased even when the ratio of cyclic stretching was $10 \%$ (Figure 3J). These results suggest that the perpendicular migration and increase in the migration speed in response to the cyclic stretching stimuli are mediated by different mechanisms.

The mechanical interaction between the cell and substratum may act as a primitive signaling cascade for determining polarity and migration direction because cells can migrate even if there are no additional environmental signals (such as a concentration gradient of a chemoattractant). It is generally believed that the mechanism of cell migration is based on actin polymerization at the front of the migrating cells, and actomyosin-dependent contraction at the rear (30). We proposed a model for cell migration in which myosin II may accumulate and exert an active force at the stretched region of the cell (31). The aspiration of a small part of the cell surface by a micropipette induced myosin II accumulation at the tip of the aspirated cell lobe, suggesting that myosin II may accumulate in response to mechanical stimulation (5). Our hypothesis of the mechanism of the migration perpendicular to the cyclic stretching is as follows: In response to the right-left cyclic stretching of the substratum, myosin II may accumulate right-left symmetrically. This may make the probabilities of the right and left migration equiprobable. A future topic of interest is determining whether the stretch stimuli generate polarized cell migration via polarized regulation of actin polymerization or actomyosin-dependent contraction. Further detailed observations using the new device described here will shed light on the relationship between the mechanosensing and molecular machinery involved in cell migration.

\section{Acknowledgments}

We thank Peter N. Devreotes (Johns Hopkins University, Baltimore, MD, USA) for the kind gift of the Dictyostelium cell line RI9. This study was partly supported by a Grant-in-Aid for Scientific Research on Priority Areas to SY from Japan's Ministry of Education, Culture, Sports, Science and Technology (MEXT). We are grateful for their support.

The authors declare no competing interests.

\section{References}

1. Chien, S. 2008. Effects of disturbed flow on endothelial cells. Ann. Biomed. Eng. 36:554562.

2. Ricci, A.J., B. Kachar, J. Gale, and S.M. Van Netten. 2006. Mechano-electrical transduction new insights into old ideas. J. Membr. Biol. 209:71-88.

3. Mitchson, J.M. and M.M. Swann. 1954. The mechanical properties of the cell surface: $I$. The cell elastimeter. J. Exp. Biol. 31:31443-31460.

4. Hochmuth, R.M. 2000. Micropipette aspiration of living cells. J. Biomech. 33:15-22.

5. Merkel, R., R. Simson, D.A. Simson, M. Hohenadl, A. Boulbitch, E. Wallraff, and E. Sackmann. 2000. A micromechanic study of cell polarity and plasma membrane cell body coupling in Dictyostelium. Biophys. J. 79:707719.

6. Effler, J.C., Y. Kee, J.M. Berk, M.N. Tran, P.A. Iglesias, and D.N. Robinson. 2006. Mitosisspecific mechanosensing and contractile-protein redistribution control cell shape. Curr. Biol. 16:1962-1967.

7. Wu, C.-C., Y.-S. Li, J.H. Haga, R. Kaunas, J.-J. Chiu, F.-C. Su, S. Usami, and S. Chien. 2007. Directional shear flow and Rho activation prevent the endothelial cell apoptosis induced by micropatterned anisotropic geometry. Proc. Natl. Acad. Sci. USA 104:1254-1259.

8. Chang, S.-F., C.A. Chang, D.-Y. Lee, P.-L. Lee, Y.-M. Yeh, C.-R. Yeh, C.-K. Cheng, S. Chien, and J.-J. Chiu. 2008. Tumor cell cycle arrest induced by shear stress: roles of integrins and Smad. Proc. Natl. Acad. Sci. USA 105:39273932.

9. Dalous, J., E. Burghardt, A. Muller-Taubenberger, F. Bruckert, G. Gerisch, and T. Bretschneider. 2008. Reversal of cell polarity and actin-myosin cytoskeleton reorganization under mechanical and chemical stimulation. Biophys. J. 94:1063-1074.

10. Fass, J.N. and D.J. Odde. 2003. Tensile forcedependent neurite elicitation via anti-beta 1 integrin antibody-coated magnetic beads. Biophys. J. 85:623-636. 
11. Tanase, M., N. Biais, and M. Sheetz. 2007. Magnetic tweezers in cell biology. Methods Cell Biol. 83:473-493.

12. Henon, S., G. Lenormand, A. Richert, and F. Gallet. 1999. A new determination of the shear modulus of the human erythrocyte membrane using optical tweezers. Biophys. J. 76:1145-1151.

13. Mills, J.P., M. Diez-Silva, D.J. Quinn, M. Dao, M.J. Lang, K.S.W. Tan, C.T. Lim, G. Milon, et al. 2007. Effect of plasmodial RESA protein on deformability of human red blood cells harboring Plasmodium falciparum. Proc. Natl. Acad. Sci. USA 104:9213-9217.

14. Lim, C.T., E.H. Zhou, and S.T. Quek. 2006. Mechanical models for living cells. J. Biomech. 39:195-216.

15. Giannone, G. and M.P. Sheetz. 2006. Substrate rigidity and force define form through tyrosine phosphatase and kinase pathways. Trends Cell Biol. 16:213-223.

16. Vogel, V. and M. Sheetz. 2006. Local force and geometry sensing regulate cell functions. Nat. Rev. Mol. Cell Biol. 7:265-275.

17. Naruse, K., T. Yamada, and M. Sokabe. 1998. Involvement of SA channels in orienting response of cultured endothelial cells to cyclic stretch. Am. J. Physiol. 274:H1532-H1538.

18. Naruse, K., T. Yamada, X.R.Sai, M. Hamaguchi, and M. Sokabe. 1998. Pp125FAK is required for stretch dependent morphological response of endothelial cells. Oncogene 17:455-463.

19. Birukov, K.G., J.R. Jacobson, A.A. Flores, S.Q. Ye, A.A. Birukova, A.D. Verin, and J.G.N. Garcia. 2003. Magnitude-dependent regulation of pulmonary endothelial cell barrier function by cyclic stretch. Am. J. Physiol. Lung Cell. Mol. Physiol. 285:L785-L797.

20. Kaunas, R., P. Nguyen, S. Usami, and S. Chien. 2005. Cooperative effects of Rho and mechanical stretch on stress fiber organization. Proc. Natl. Acad. Sci. USA 102:15895-15900.

21. Mark, J.E. 1999. Polymer Data Handbook. Oxford University Press, New York.

22.Insall, R.H., R.D. Soede, P. Schaap, and P.N. Devreotes. 1994. Two cAMP receptors activate common signaling pathways in Dictyostelium. Mol. Biol. Cell 5:703-711.

23. Li, L., S.F. Nørrelykke, and E.C. Cox. 2008. Persistent cell motion in the absence of external signals: A search strategy for eukaryotic cells. PLoS One 3:e2093.

24. Lazopoulos, K.A. and D. Stamenovi. 2008. Durotaxis as an elastic stability phenomenon. J. Biomech. 41:1289-1294.

25.Jiang, G., A.H. Huang, Y. Cai, M. Tanase, and M.P. Sheetz. 2006. Rigidity sensing at the leading edge through alphavbeta 3 integrins and RPTPalpha. Biophys. J. 90:1804-1809.

26. Lo, C.M., H.B. Wang, M. Dembo, and Y.L. Wang. 2000. Cell movement is guided by the rigidity of the substrate. Biophys. J. 79:144-152.

27. Andrew, N. and R.H. Insall. 2007. Chemotaxis in shallow gradients is mediated independently of PtdIns 3-kinase by biased choices between random protrusions. Nat. Cell Biol. 9:193-200.

28.Sato, M.J., H. Kuwayama, W.N. van Egmond, A.L.K. Takayama, H. Takagi, P.J.M. van Haastert, T. Yanagida, and M. Ueda. 2009. Switching direction in electric-signal-induced cell migration by cyclic guanosine monophosphate and phosphatidylinositol signaling. Proc. Natl Acad. Sci. USA 106:6667-6672.

29. Pullar, C.E., B.S. Baier, Y. Kariya, A.J. Russell, B.A. Horst, M.P. Marinkovich, and R.R. Isseroff. 2006. Beta4 integrin and epidermal growth factor coordinately regulate electric fieldmediated directional migration via Rac1. Mol. Biol. Cell 17:4925-4935.

30. Yumura, S., H. Mori, and Y. Fukui. 1984 Localization of actin and myosin for the study of ameboid movement in Dictyostelium using improved immunofluorescence. J. Cell Biol. 99:894-899.

31. Iwadate, Y. and S. Yumura. 2008. Actin-based propulsive forces and myosin-II-based contractile forces in migrating Dictyostelium cells. J. Cell Sci. 121:1314-1324.

Received 19 April 2009; accepted 10 July 2009.

Address correspondence to Yoshiaki Iwadate, Department of Functional Molecular Biology, Graduate School of Medicine, Yamaguchi University, Yamaguchi 753-8512, Japan. e-mail iwadate@yamaguchi-u.ac.jp

\section{BioTechniques \\ The International Journal of Life Science Methods}

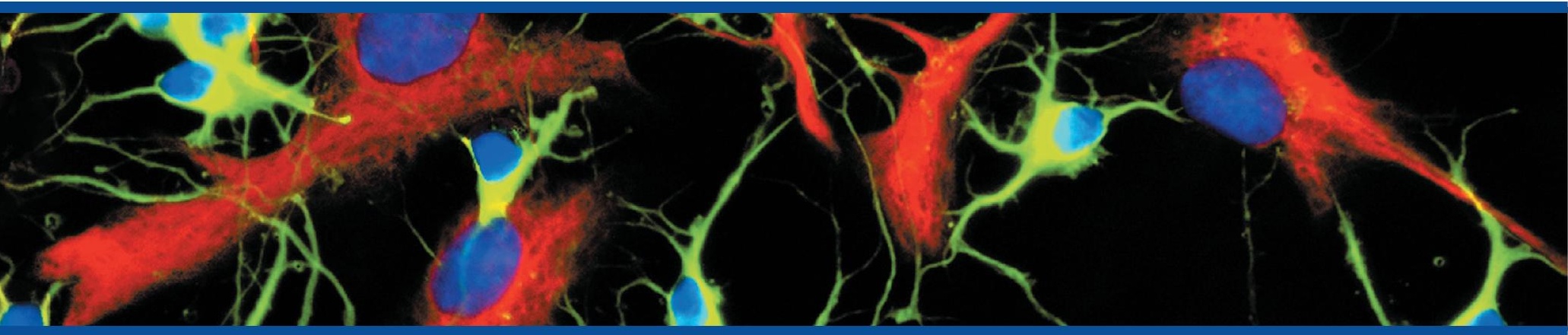

BioTe BioTechniques int $>0$

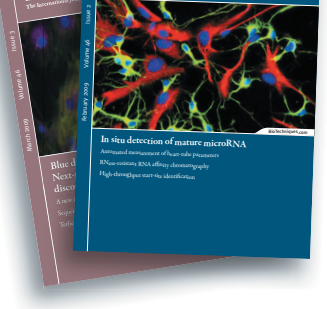

\section{Update your subscription}

Update your profile today to ensure that your copy of BioTechniques experiences no delivery disruptions.

Go to:

www.BioTechniques.com/subscribe 